\title{
Chiral thiourea derivatives as organocatalyts in the enantioselective Morita- Baylis-Hillman reactions
}

\author{
A. Ebru Aydin \\ Hatay Mustafa Kemal University \\ Department of Chemistry, Tayfur Sokmen Campus 31040 \\ Hatay, Turkey \\ E-mail: aydin@mku.edu.tr
}

Received mm-dd-yyyy

Accepted mm-dd-yyyy

Published on line mm-dd-yyyy

Dates to be inserted by editorial office

\section{Abstract}

Novel chiral bifunctional thiourea derivatives have been synthesised and successfully applied to the intermolecular Morita-Baylis-Hillman (MBH) reaction of an aromatic aldehyde with methyl vinyl ketone (MVK) and to the intramolecular $\mathrm{MBH}$ reaction of $\omega$-formyl-enone. The corresponding products were obtained with high enantioselectivities (up to $98 \%$ ee). The thiourea organocatalyst derived from a 8 -amino alcohol gave high enantioselectivities ( $92 \%$ ee) in the intermolecular $\mathrm{MBH}$ reaction, whereas the same chiral ligand afforded the corresponding product in high yield (85\%) with moderate enantioselectivity $(75 \%$ ee) in the intramolecular $\mathrm{MBH}$ reaction. The use of a thiophene ring-containing thiourea derivative gave high enantioselectivities in the intermolecular and intramolecular $\mathrm{MBH}$ reactions ( $85 \%$ and $95 \%$ ee, respectively).

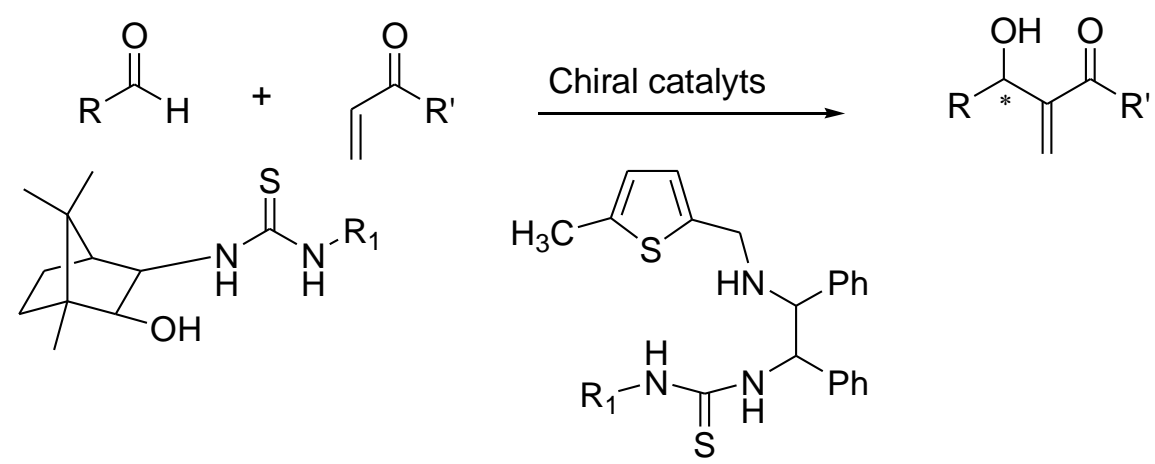

Keyword: Intermolecular Morita-Baylis-Hillman reaction, intramolecular Morita-Baylis-Hillman reaction reaction; 8 -amino alcohol; chiral thiourea derivatives 


\section{Introduction}

The Morita-Baylis-Hillman (MBH) reaction is one of the most important methods for forming $\mathrm{C}-\mathrm{C}$ bonds ${ }^{1-3}$. It involves the coupling of electron-deficient alkenes with $\mathrm{sp}^{2}$ hybridised carbon electrophiles, such as aldehydes, ketones and aldimines and is catalysed by nucleophilic amines or phosphines. ${ }^{4}$ Various activated alkenes such as enals, ${ }^{5,6}$ enones, ${ }^{7-9}$ acrylates ${ }^{10,11}$ and acrylamides ${ }^{12,13}$ have been successfully used in the MBH reaction.

Morita ${ }^{14}$ described a novel reaction between various aldehydes and acrylic compounds catalysed by a tertiary phosphine (tricyclohexylphosphine) and yielding vinylic compounds. Subsequently, in 1972, Baylis and Hillman ${ }^{15}$ reported a similar reaction between acetaldehyde and ethyl acrylate or acrylonitrile. Instead of phosphines, they used 1,4- diazabicyclo[2.2.2]octane (DABCO) as a Lewis base to obtain products similar to those of Morita's study (Scheme 1).

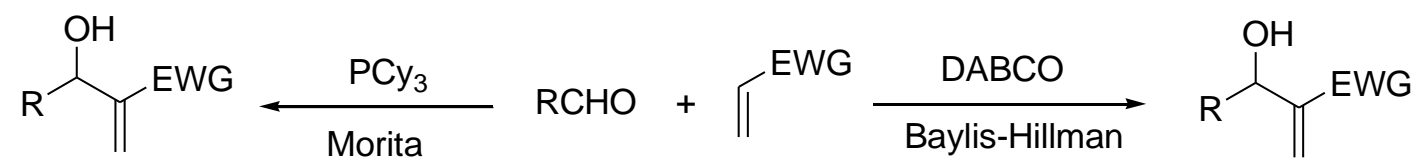

Scheme 1. The Morita-Baylis-Hillman reaction.

Since then, various chiral catalysts such as chiral Lewis acid or Brønsted-Lowry acid catalysts, ${ }^{16-18}$ chiral amino- or phosphino-type catalysts, ${ }^{19-21}$ chiral amino acid derivatives, ${ }^{22,23}$ a chiral thiourea-type catalyst ${ }^{24}$ and chiral ionic liquids ${ }^{25}$ have been reported for this asymmetric catalytic process. These chiral catalysts have been developed for intermolecular MBH reactions. ${ }^{26}$ Optically active $\beta$-hydroxy- $\alpha$-methylene carbonyl compounds are useful intermediates in natural product synthesis.

To synthesise these compounds, several enantioselective versions of the $\mathrm{MBH}$ reaction involving a chiral catalyst have been reported. ${ }^{27}$ Barrett et al. ${ }^{28}$ used chiral bicyclic pyrrolizidine derivatives (A) as asymmetric catalysts for the $\mathrm{MBH}$ reaction of ethyl vinyl ketone and an aromatic aldehyde. Hatakeyama et al. ${ }^{29}$ used 6 -isocupreidine (B) to catalyse the asymmetric $\mathrm{MBH}$ reaction between aldehydes and the strongly activated Michael acceptor 1,1,1,3,3,3-hexa-fluoroisopropyl acrylate (HFIP). Hayashi et al. ${ }^{30}$ developed chiral diamines (C) as catalysts for the MBH reaction of MVK and electron-deficient benzaldehyde, affording adducts with enantioselectivities up to $75 \%$ (Scheme 2). 
<smiles>[R]C(=O)C=CC(=O)C([R])O</smiles>

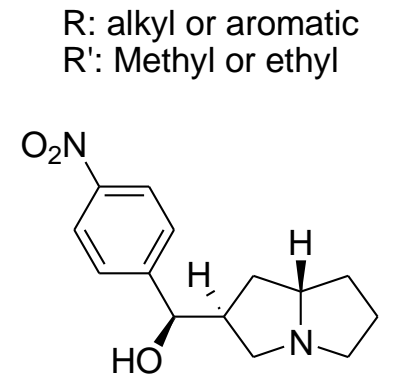

$21-72 \%$ ee

A<smiles>CC(C)[C@H](Cc1ccccc1)NC(=S)Nc1cc(C(F)(F)F)cc(C(F)(F)F)c1</smiles>

up to $83 \%$ ee<smiles>CCC12CCN3CC(CC31)O[C@H]2c1ccnc2ccc(O)cc12</smiles>

91->99\% ee

B

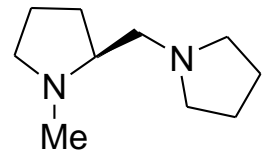

up to $75 \%$ ee

C

D

Scheme 2. Enantioselective intermolecular $\mathrm{MBH}$ reaction

Chiral thiourea derivatives such as amino alcohol-derived thiourea and bifunctional phosphinothiourea have been used successfully for the intermolecular $\mathrm{MBH}$ reaction. ${ }^{31-33}$ In the presence of $L$-valine-derived phosphinothiourea (D), the MBH products were obtained in good enantioselectivities (up to 83\% ee) (Scheme 2). ${ }^{34}$ The intramolecular $\mathrm{MBH}$ reaction can be performed when suitably oriented electrophilic and activated alkene moieties are present in the same molecule, but few examples of intramolecular $\mathrm{MBH}$ reactions are found in the literature.

In 1992, Franter and co-workers reported the first asymmetric intramolecular MBH reaction using $p$ chiral phosphine (-)-CAMP to catalyse cyclisation of an enoate onto a ketone to afford cyclopenten-ol ester in $14 \%$ ee. $^{35-37}$

Pipecolinic acid (E) and $N$-methylimidazole were used by Miller as a cocatalytic system to catalyse an intermolecular $\mathrm{MBH}$ reaction (84\% ee). ${ }^{38}$ (Scheme 3 ). 

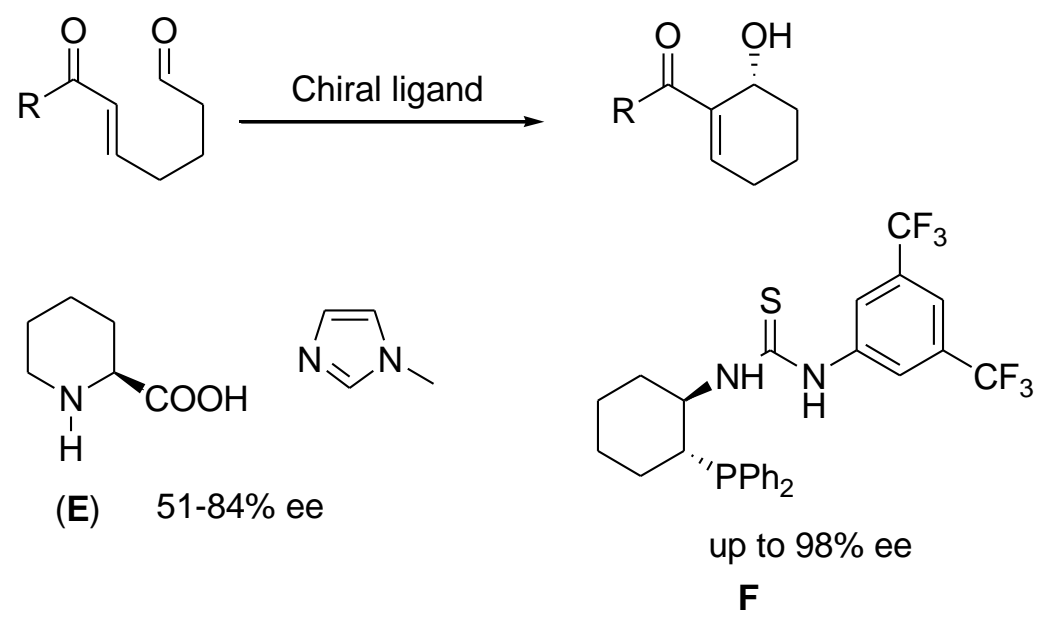

Scheme 3. Enantioselective intramolecular $\mathrm{MBH}$ reaction

Wu et al. ${ }^{39}$ developed chiral cyclohexane-based (F) and amino acid-derived phosphinothioureas as catalysts for the enantioselective intramolecular $\mathrm{MBH}$ reaction of $\omega$-formyl- $\alpha$, $\beta$-unsaturated carbonyl compounds with up to $98 \%$ and $84 \%$ ee, respectively (Scheme 3 ). Honh et al. ${ }^{40}$ reported the proline-catalysed intermolecular $\mathrm{MBH}$ reactions of hept-2-enedial and the corresponding products were obtained in high enantiomeric excess (98\% ee) and good yield (74\%). Gladysz et al. ${ }^{41}$ reported an intramolecular $\mathrm{MBH}$ reaction in the presence of rhenium-containing phosphine $\left(\eta^{5}-\mathrm{C}_{5} \mathrm{H}_{5}\right) \operatorname{Re}(\mathrm{NO})\left(\mathrm{PPh}_{3}\right)\left(\mathrm{CH}_{2} \mathrm{PAr}_{2}\right)$, affording the product in $74 \%$ ee.

In this study, the intermolecular $\mathrm{MBH}$ reaction of an aromatic aldehyde with methyl vinyl ketone (MVK) and the intramolecular $\mathrm{MBH}$ reaction of $\omega$-formyl-enone catalysed by the thioureas $7 \mathbf{a}-\mathbf{d}$ and $\mathbf{1 1 a - d}$ are presented.

\section{Results and Discussion}

The synthesis of chiral thiourea derivatives 7a-d. The synthesis of $\beta$-amino alcohol 6 from (1S)-(-)-camphor was performed according to the literature procedure. ${ }^{42,43}$ Chiral amino alcohol-based thioureas $7 a-\mathbf{d}$ were easily obtained by condensation of $\beta$-amino alcohol 6 with 1.1 equiv. of the corresponding isothiocyanate in $\mathrm{CH}_{2} \mathrm{Cl}_{2}$ at room temperature (Scheme 4 ).

Chiral diamine 10 was prepared in a two-step reaction involving condensation of $(1 S, 2 S)-1,2-$ diphenylethane-1,2-diamine 9 with 1 equiv. of 5-methyl thiophene-2-carbaldehyde 8 , followed by reduction with $\mathrm{LiAlH}_{4}$.

For the synthesis of $\mathbf{7 a - d}$ and $\mathbf{1 1 a - d , ~ c h i r a l ~ c o m p o u n d s ~} \mathbf{6}$ and $\mathbf{1 0}$ were stirred with 1.1 equiv. of the corresponding isothiocyanates at room temperature (Scheme 4). 


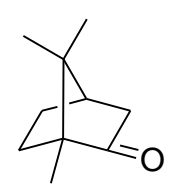

4

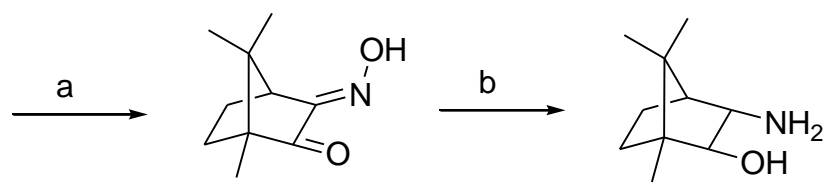

5

a: $\mathrm{R}_{1}=\mathrm{C}_{6} \mathrm{H}_{5}$

b: $\mathrm{R}_{1}=3,5-\left(\mathrm{CF}_{3}\right)_{2} \mathrm{C}_{6} \mathrm{H}_{3}$

c: $\mathrm{R}_{1}=4-\mathrm{ClC}_{6} \mathrm{H}_{4}$

d: $\mathrm{R}_{1}=4-\mathrm{MeoC}_{6} \mathrm{H}_{4}$ (2-exo-3-exo)-6

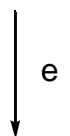

(2-exo-3-exo)-7<smiles>Cc1ccc(C=O)s1</smiles>

8<smiles>NC(c1ccccc1)C(N)c1ccccc1</smiles>

9<smiles>Cc1ccc(CNC(c2ccccc2)C(N)c2ccccc2)s1</smiles>

10<smiles>[R8]NC(=S)NC(c1ccccc1)C(NCc1ccc(C)s1)c1ccccc1</smiles>

Scheme 4. Synthetic route of the chiral thiourea derivatives

The enantioselective MBH reaction of $p$-nitrobenzaldehyde with methyl vinyl ketone (MVK).

To determine the best catalyst, the model reaction of $p$-nitrobenzaldehyde and MVK was initially performed in the presence of 10 mol\% of catalysts $\mathbf{7}$ and 11a-d, and the results are summarised in Table 1, (Scheme 5) .<smiles>C=CC(C)=O</smiles>

1<smiles>O=Cc1ccc([N+](=O)[O-])cc1</smiles>

2
Chiral catalyts<smiles>C=C(C(C)=O)[C@H](O)c1ccc([N+](=O)[O-])cc1</smiles>

Scheme 5. Enantioselective intermolecular $\mathrm{MBH}$ reaction

Chiral ligand screening showed that $\beta$-amino alcohol-based thiourea 7a provided the corresponding product in high chemical yield (80\%) with $60 \%$ ee. The use of $7 \mathbf{b}$ as a chiral ligand-bearing strong electronwithdrawing substituent on the phenyl group afforded $\mathrm{MBH}$ adduct 3a in $78 \%$ yield with $80 \%$ ee. In the presence of $\mathbf{7 c}$ or $\mathbf{7 d}$ as the chiral ligand, the corresponding $\mathrm{MBH}$ product $3 \mathbf{3 a}$ was obtained in $75 \%$ chemical yield with $70 \%$ ee and $78 \%$ chemical yield with $65 \%$ ee, respectively (Table 1 , entries 3,4 ). In the presence 11a-d, good enantioselectivities and chemical yields were obtained (Table 1, entries 5-8). Performing the MBH reaction with 11 a gave the corresponding product in $78 \%$ yield with $72 \%$ ee. The use of $\mathbf{1 1 b}$ as the chiral ligand afforded the corresponding product in $77 \%$ yield with $74 \%$ ee. 
The enantioselectivity decreased with an increase in the reaction time from 30 to $60 \mathrm{~min}$. In the presence of $7 \mathrm{a}$, a higher conversion ( $85 \%$ yield) was obtained after $60 \mathrm{~min}$, but the enantioselectivity decreased $(72 \%$ ee) (entry 11$)$. The $\mathrm{MBH}$ product after 45 min was obtained in $78 \%$ chemical yield with $92 \%$ ee. The absolute configuration of the intermolecular $\mathrm{MBH}$ products, $R$, was assigned by comparing the retention time (chiral HPLC) with its literature values. ${ }^{23,30,31,33,48}$

Table 1. Screening of the chiral ligands for the $\mathrm{MBH}$ reaction of $p$-nitrobenzaldehyde with methyl vinyl ketone $(\mathrm{MVK})^{\mathrm{a}}$

\begin{tabular}{ccccc}
1 & & & \\
\hline
\end{tabular}

Because 7a gave the highest enantioselectivity in the $\mathrm{MBH}$ reaction of $p$-nitrobenzaldehyde with MVK, it was identified as most effective among the chiral ligand series (Table 1, entry 10) and was selected for optimising further the reaction conditions.

Then, the solvent effect on the $\mathrm{MBH}$ reaction of $p$-nitrobenzaldehyde with MVK was investigated using $7 \mathrm{a}$ as the chiral ligand. The results, outlined in Table 2, show that the highest enantiomeric excess (92\%) was observed in $\mathrm{CH}_{2} \mathrm{Cl}_{2}$. In polar solvents such as $\mathrm{EtOH}, \mathrm{DMF}$ and $\mathrm{MeCN}$, the $\mathrm{MBH}$ adduct 3a was obtained in low 
yield and ee (Table 2, entries 5-7). The lowest enantioselectivity was obtained in DMSO. Increasing the reaction temperature from room temperature to $40{ }^{\circ} \mathrm{C}$ decreased the ee value to $77 \%$ (Table 2 , entries 4,9 ), whereas decreasing the reaction temperature to $-15{ }^{\circ} \mathrm{C}$ obtained the corresponding product in $45 \%$ yield with $65 \%$ ee(Table 2 , entry 10 ).

Table 2. Effects of the solvents and reaction temperature on the $\mathrm{MBH}$ reaction of $p$-nitrobenzaldehyde with $\mathrm{MVK}^{\mathrm{a}}$

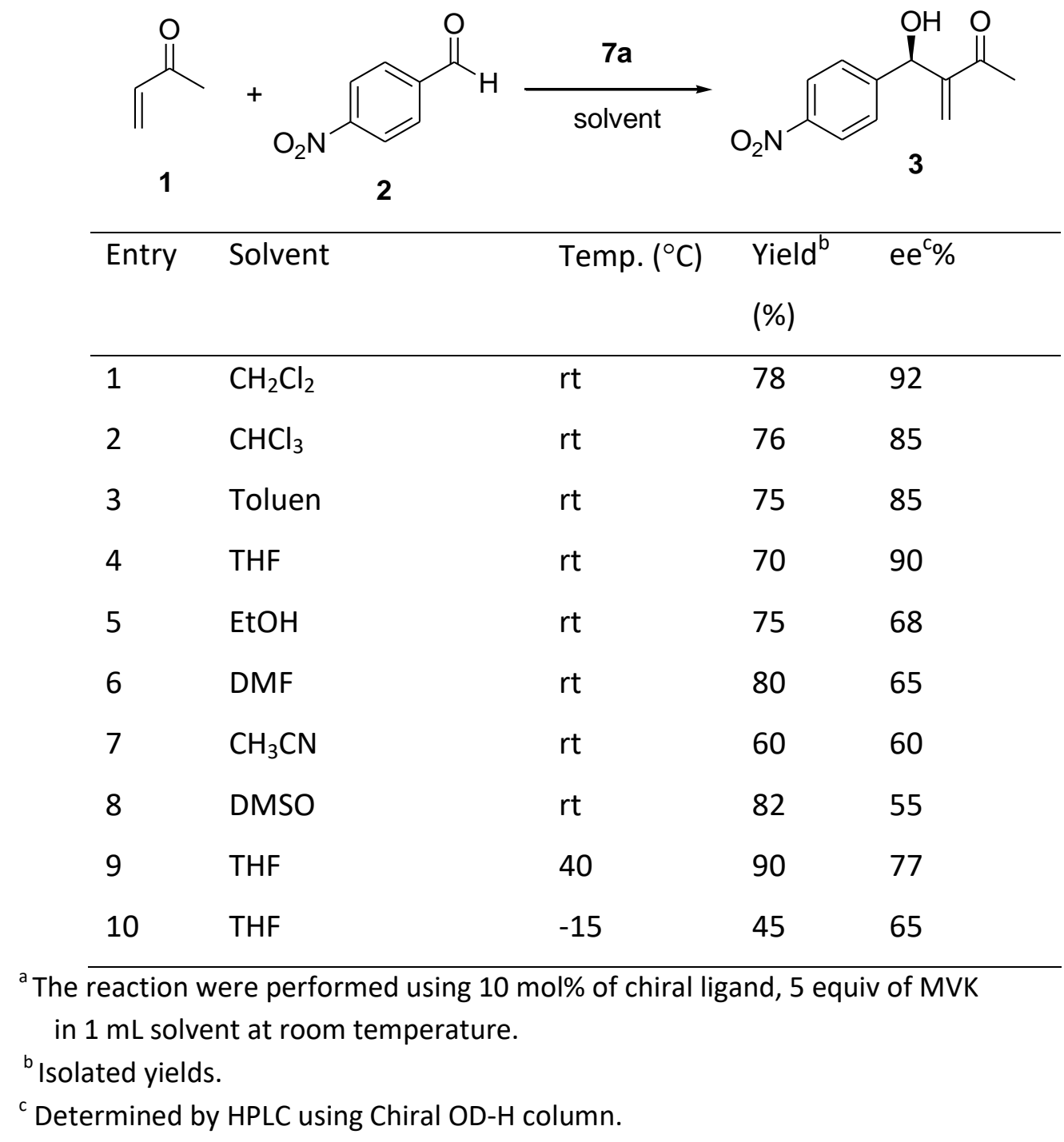

To investigate the effect of electron-withdrawing and electron-donating substituents, the asymmetric $\mathrm{MBH}$ reaction was performed with different aromatic aldehydes using conditions optimised for ligand $7 a$ (10 mol\% of $7 a$ catalyst, 5 equiv. of $\mathrm{MVK}, \mathrm{CH}_{2} \mathrm{Cl}_{2}$ as a solvent, room temperature). The results are summarised in Table 3. The electronic effects on the aromatic ring of the aldehyde affected the reactivity and selectivity. Electron-withdrawing substituents gave higher enantioselectivities than did electron-donating substituents. It is known that the electrophilicity of the carbonyl carbon atom in aryl aldehydes is increased by electronwithdrawing groups and decreased by electron-donating groups; therefore, the substrates with an electronwithdrawing substituent are expected to afford a faster reaction, leading to higher enantioselectivity. In fact, a lower enantioselectivity was observed for the meta-substituted benzaldehyde compared to that of its relevant 
para-substituted analogue (entry 3 and 4). With ligand 7a, the lowest enantiomeric excesses were observed for aromatic aldehydes bearing ortho substituents, probably due to the ortho effects (entry 2 and 7). In the presence of ligand $7 \mathrm{a}$, the non-substituted benzaldehyde afforded the product with $85 \%$ ee but in low yield (62\%). The yields and ee values decreased (62\% yield, 55\% ee) when the methoxy group on the phenyl ring was at the para position(entries 10).

Table 3. The enantioselective $\mathrm{MBH}$ reaction involving various substitutes aldeyheds ${ }^{\mathrm{a}}$

\begin{tabular}{llll} 
& & \\
\hline
\end{tabular}

The enantioselective intramolecular MBH reaction of $\omega$-formyl-enone catalyzed by $\beta$-amino alcohol-based thiourea 7 and 11a-d.

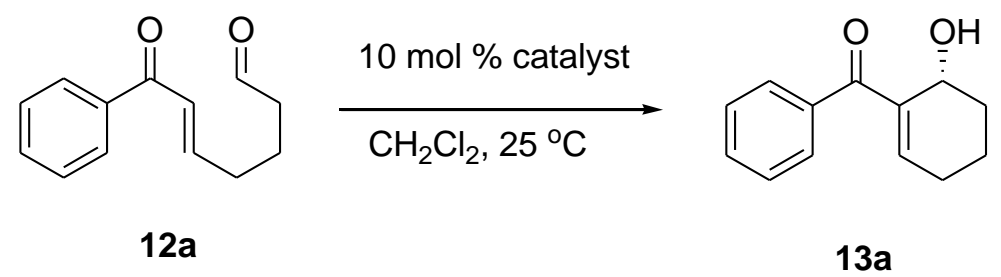

Scheme 6. Enantioselective intramolecular $\mathrm{MBH}$ reaction 
Chiral thiourea derivatives 7 and 11a-d were examined in the enantioselective intramolecular $\mathrm{MBH}$ reaction of $\omega$-formyl-enone as the model substrate (Scheme 6 ). $\beta$-Amino alcohol-based thioureas $7 a$ were used as catalysts in the intramolecular reaction of substrate $12 \mathrm{a}$ in $\mathrm{CH}_{2} \mathrm{Cl}_{2}$ at room temperature, and the $\mathrm{MBH}$ product 13 a was obtained in $65 \%$ chemical yield with good enantioselectivity ( $72 \%$ ee). As shown in Table 4 , the structure of the chiral ligands significantly affected the enantioselectivity and the chemical yield. The thiourea moiety of the chiral ligands played an important role in obtaining a high yield and enantioselectivity. Chiral thiourea derivatives $\mathbf{7 b}$ and $\mathbf{1 1} \mathbf{b}$, bearing strong electron-withdrawing substituents at the phenyl ring, afforded the corresponding product in $63 \%$ chemical yield with $95 \%$ ee and in $65 \%$ chemical yield with $94 \%$ ee, respectively. Chiral ligand $\mathbf{7 b}$ provided the corresponding product in higher enantioselectivity and chemical yield than ligands $\mathbf{7 a , c , d}$ did (Table 4, entries 1-4). These observations were agreement with the observations of Wu et $a l^{39}$ The absolute configuration of the intramolecular $\mathrm{MBH}$ products is $R$-configuration, which was assigned by comparing the retention time (chiral HPLC) with those reported in the literatures. ${ }^{30,39,41,48,46}$

Table 4. Catalysts screening for the intramolecular $\mathrm{MBH}$ reaction of $12 \mathrm{a}^{\mathrm{a}}$

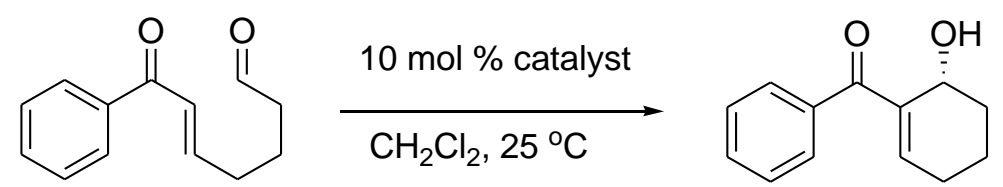

$12 a$

$13 a$

\begin{tabular}{llll}
\hline Entry & Catalyst (yield\%) & Yield $^{\mathrm{b}}(\%)$ & $\mathrm{ee}^{\mathrm{c}}(\%)$ \\
\hline 1 & 7a & 65 & 72 \\
2 & 7b & 63 & 95 \\
3 & 7c & 74 & 65 \\
4 & 7d & 73 & 60 \\
5 & 11a & 67 & 68 \\
6 & 11b & 65 & 94 \\
7 & 11c & 76 & 75 \\
8 & 11d & 73 & 65
\end{tabular}

${ }^{a}$ The reaction were performed using 10 mol\% of chiral ligand, 5 equiv of $\omega$-formyl-enone in $1 \mathrm{~mL}$ solvent at room temperature, $45 \mathrm{~min}$.

${ }^{\mathrm{b}}$ Isolated yields.

${ }^{c}$ Determined by HPLC using Chiral OD-H column.

Because $\mathbf{7 b}$ gave the highest enantioselectivity in the intramolecular $\mathrm{MBH}$ reaction, it was identified as most effective among the chiral ligand series (Table 4, entry 2) and was selected for optimising further the reaction conditions.

To further improve the enantioselectivity of $12 a$, the effect of solvent on the enantioselective intramolecular $\mathrm{MBH}$ reaction of $\omega$-formyl-enone was investigated (Table 5, entries 1-8). The enantioselective intramolecular $\mathrm{MBH}$ reaction was performed in solvents such as $n$-hexane, toluene, $\mathrm{CHCl}_{3}, \mathrm{CH}_{2} \mathrm{Cl}_{2}, \mathrm{THF}$, 
acetone, $\mathrm{CH}_{3} \mathrm{CN}, \mathrm{DMF}, \mathrm{MeOH}$ and $\mathrm{EtOH}$. Table 5 shows that $\mathrm{CH}_{2} \mathrm{Cl}_{2}$ was the most appropriate solvent (entry 4). In $\mathrm{DMF}$, the corresponding product was obtained in $10 \%$ chemical yield with $25 \%$ ee (entry 8 ). In $\mathrm{MeOH}$, the $\mathrm{MBH}$ product $13 \mathrm{a}$ was obtained in moderate chemical yield (72\%) but with poor enantioselectivity (12\% ee) (entry 9).

Table 5. The effect of the solvents on the intramolecular $\mathrm{MBH}$ reaction ${ }^{\mathrm{a}}$

\begin{tabular}{llll} 
& \\
\hline & & & \\
\hline
\end{tabular}

The enantioselective intramolecular $\mathrm{MBH}$ reaction involving various $\omega$-formyl-enone substrates was investigated, and the results are summarised in Table 6. All the substituted aromatic enones were converted to the $\mathrm{MBH}$ reaction product in 62-95\% ee. As shown in Table 6, the substrates with an electron-withdrawing substituent at the para position of the phenyl ring afforded higher enantioselectivity than those with an electron-donating substituent or without a phenyl substituent.

With a substrate-bearing substituent at the ortho position of the phenyl group, the corresponding product was obtained in excellent yield with poor enantioselectivity, due to the ortho effect (entries 2 and 8 ). 
Table 6. The enantioselective intramolecular $\mathrm{MBH}$ reaction involving various $\omega$-formyl-enone substrates ${ }^{\mathrm{a}}$

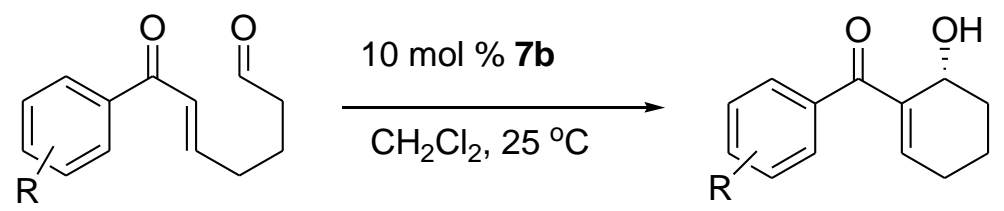

12

13

\begin{tabular}{|c|c|c|c|}
\hline Entry & $\mathrm{Ar}$ & Yield $^{\mathrm{b}}(\%)$ & $\mathrm{ee}^{\mathrm{c}}(\%)$ \\
\hline 1 & $\mathrm{C}_{6} \mathrm{H}_{5}, 12 \mathrm{a}$ & 87 & 83 \\
\hline 2 & $4-\mathrm{NO}_{2}-\mathrm{C}_{6} \mathrm{H}_{4}, \mathbf{1 2 b}$ & 63 & 95 \\
\hline 3 & $2-\mathrm{Br}-\mathrm{C}_{6} \mathrm{H}_{4}, 12 \mathrm{c}$ & 90 & 75 \\
\hline 4 & $3-\mathrm{Br}-\mathrm{C}_{6} \mathrm{H}_{4}, \mathbf{1 2 d}$ & 90 & 78 \\
\hline 5 & $4-\mathrm{Br}-\mathrm{C}_{6} \mathrm{H}_{4}, \mathbf{1 2 e}$ & 85 & 80 \\
\hline 6 & $4-\mathrm{Cl}^{-} \mathrm{C}_{6} \mathrm{H}_{4}, \mathbf{1 2 f}$ & 83 & 81 \\
\hline 7 & $4-\mathrm{F}-\mathrm{C}_{6} \mathrm{H}_{4}, 12 \mathrm{~g}$ & 78 & 80 \\
\hline 8 & $2-\mathrm{Me}-\mathrm{C}_{6} \mathrm{H}_{4}, \mathbf{1 2 h}$ & 95 & 62 \\
\hline 9 & 4-Me- $\mathrm{C}_{6} \mathrm{H}_{4}, \mathbf{1 2 j}$ & 80 & 65 \\
\hline 10 & 4-MeO- $\mathrm{C}_{6} \mathrm{H}_{4}, \mathbf{1 2} \mathbf{j}$ & 78 & 60 \\
\hline 11 & Thiophen-2-yl, 12k & 65 & 72 \\
\hline
\end{tabular}

\footnotetext{
a The reaction were performed using 10 mol\% of chiral ligand, 5 equiv of $\omega$-formyl-enone in $1 \mathrm{~mL} \mathrm{CH} \mathrm{Cl}_{2}$ at room temperature.

${ }^{\mathrm{b}}$ Isolated yields.

${ }^{c}$ Determined by HPLC using Chiral OD-H column.
}

\section{Conclusions}

In summary, we have developed the $\mathrm{MBH}$ reaction of an aromatic aldehyde with MVK and the intramolecular $\mathrm{MBH}$ reaction of $\omega$-formyl-enone catalysed by thioureas $7 \mathbf{a}-\mathbf{d}$ and $\mathbf{1 1 a - d}$. The reaction proceeds under very mild conditions to quickly afford the desired product in good to excellent yields with generally excellent enantiomeric excesses. The use of $(1 R, 2 S)-7 \mathbf{b}$ as a chiral ligand bearing a strong electron-withdrawing substituent at the phenyl group afforded the hydroxyl enone with low enantioselectivity (40 \% ee) in the intermolecular $\mathrm{MBH}$ reaction, whereas the same ligand gave the highest enantioselectivity ( $97 \%$ ee) in the intramolecular $\mathrm{MBH}$ reaction. Thiophene ring-containing thiourea derivatives bearing a strong electronwithdrawing substituent on the phenyl group 11b gave a high enantioselectivity in the intermolecular $\mathrm{MBH}$ reaction $(85 \%$ ee), whereas the same ligand afforded the cyclic hydroxyl enones with excellent enantioselectivity ( $94 \%$ ee). The application of these ligands to the other asymmetric reactions is now under investigation. 


\section{Experimental Section}

General. Reagents and solvents were purchased from Aldrich, Merck and Fluka. All solvents were dried before use according to the standard procedures. All reactions were carried out under Ar atmosphere and monitored by thin-layer chromatography (TLC) on Merck silica gel plates (60 F-254) using UV light or phosphomolybdic acid in methanol. E. Merck silica gel (60, particle size $0.040-0.063 \mathrm{~mm}$ ) was used for flash chromatography. All NMR spectra were recorded on a Bruker DPX-400 MHz spectrometer at room temperature. Chemical shifts (parts per million) are reported relative to TMS. Coupling constant are expressed as J values in Hertz. Thin layer chromatography (TLC) was performed using Merck Kieselgel 60 F254. Elemental analyses (C, H, N, S) were carried out on a Carlo Erba Model Thermo Scıentıfıc Flash 2000 elemental analyzer. Optical rotations were recorded on a Autopol IV polarimeter. All melting points were measured with an Electrothermal melting point instrument. Elemental analyses were carried out on a LECO CHNS-932 series analyzer. Enantiomeric excesses were determined by HPLC analysis using a Shimadzu and Thermo Finnigan analyzer.

Synthesis of chiral catalysts 7a-d. Camphor was converted to 3-hydroxyimino-2-oxo-1,7,7trimethylbicyclo[2.2.1] heptane 5 using ter-butyl nitrite dry THF in the presence of ter-sodium butylate ${ }^{42,43}$. 3-Amino-2-hydroxy-1,7,7-trimethylbicyclo[2.2.1] heptane 6 was obtained from the reduction of 5 with $\mathrm{LiAlH}_{4}$ by refluxing in dry ether with $79 \%$ yield, according to literature procedure. ${ }^{42-45}$

$\beta$-Amino alcohol $6(1.0 \mathrm{mmol})$ was dissolved in $\mathrm{CH}_{2} \mathrm{Cl}_{2}(2.0 \mathrm{~mL})$ was added the corresponding solution of isothiocyanate $(1.1 \mathrm{mmol})$ in $\mathrm{CH}_{2} \mathrm{Cl}_{2}(2.0 \mathrm{~mL})$ at the room temperature. The reaction mixture was allowed to stir at same temperature until reaction completed (monitoring by TLC). The solvent removed under reduced pressure and the the residue was purified by column chromatography (petroleum ether/ethyl acetate).

1-1(3-Hydroxy-4,7,7-trimethylbicyclo[2.2.1]heptan-2yl)-3-phenylthiourea (7a). White solid; 69\% yield; mp $172-174{ }^{\circ} \mathrm{C},[\alpha]_{\mathrm{D}}^{25}=-1.28\left(\mathrm{c}=0.32, \mathrm{CHCl}_{3}\right) ;{ }^{1} \mathrm{HNMR}:\left(400 \mathrm{MHz}, \mathrm{CDCl}_{3}\right) \delta 0.81(\mathrm{~s}, 3 \mathrm{H}), 0.98(\mathrm{~s}, 3 \mathrm{H}), 1.09(\mathrm{~s}, 3 \mathrm{H})$, 1.24-1.31 (m, 2H), 1.42-1.47 (m, 1H), $1.64(\mathrm{~d}, J 4.23 \mathrm{~Hz}, 1 \mathrm{H}), 1.67-1.69(\mathrm{~m}, 1 \mathrm{H}), 1.83-1.88(\mathrm{~m}, 1 \mathrm{H}), 2.65(\mathrm{~d}, J 7.2$ $\mathrm{Hz}, 1 \mathrm{H}), 3.47(\mathrm{~d}, J 7.3 \mathrm{~Hz}, 1 \mathrm{H}), 6.45-7.12(\mathrm{~m}, 5 \mathrm{H}) ;{ }^{13} \mathrm{C}-\mathrm{NMR}:\left(100 \mathrm{MHz}, \mathrm{CDCl}_{3}\right)$ 11.23, 21.36, 21.99, 27.29, 32.48, $46.91,48.79,52.28,65.49,78.48,126.12-130.23,138.32,182.25$. Anal. Calcd. for $\mathrm{C}_{17} \mathrm{H}_{24} \mathrm{~N}_{2} \mathrm{OS} \mathrm{C}, 67.07 ; \mathrm{H}, 7.95$; N, 9.20; S, 10.53; found: C, 67.12; H, 7.91; N, 9.22; S, 10.52 .

(1-(3,5-Bis(trifluormethyl)phenyl)-3-(3-hydroxy-4,7,7-trimethylbicyclo[2.2.1]heptan-2yl)urea (7b). White solid; 78\% yield; $\mathrm{mp} 180-182-{ }^{\circ} \mathrm{C},[\alpha]_{\mathrm{D}}^{25}=-1.19\left(\mathrm{c}=0.482, \mathrm{CHCl}_{3}\right){ }^{1}{ }^{1} \mathrm{HNMR}:\left(400 \mathrm{MHz}, \mathrm{CDCl}_{3}\right) \delta 0.83(\mathrm{~s}, 3 \mathrm{H}), 1.12(\mathrm{~s}$, $3 \mathrm{H}), 1.14(\mathrm{~s}, 3 \mathrm{H}), 1.45-1.49(\mathrm{~m}, 2 \mathrm{H}),, 1.65(\mathrm{~d}, J 4.1 \mathrm{~Hz}, 1 \mathrm{H}), 1.69-1.72(\mathrm{~m}, 1 \mathrm{H}), 1.84-1.91(\mathrm{~m}, 1 \mathrm{H}), 2.91(\mathrm{~d}, J 7.3$ $\mathrm{Hz}, 1 \mathrm{H}$ ), 3.59 (d, J $7.3 \mathrm{~Hz}, 1 \mathrm{H}), 7.12$ (broad s, 2H), 7.27 (s, Ar-H, 1H),; ${ }^{13} \mathrm{C}-\mathrm{NMR}:\left(100 \mathrm{MHz}, \mathrm{CDCl}_{3}\right)$ 11.26, 21.48, 22.09, 27.35, 32.56, 47.28, 48.92, 52.67, 65.78, 78.89, 125.67, 126.8, 127.12, 132.14, 138.19, 182.23 Anal. Calcd. for $\mathrm{C}_{19} \mathrm{H}_{22} \mathrm{~F}_{6} \mathrm{~N}_{2} \mathrm{OS} \mathrm{C}, 51.81 ; \mathrm{H}, 5.03 ; \mathrm{N}, 6.36 ; \mathrm{S}, 7.28$; found: C, 51.86; H, 5.09; N, 6.34; S, 7.29.

1-(4-chlorophenyl)-3-(3-hydroxy-4,7,7-trimethylbicyclo[2.2.1] heptan-2yl)thiourea (7c). White solid; 64\%

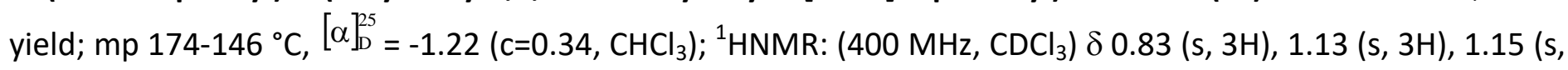
$3 \mathrm{H}), 1.47-1.51(\mathrm{~m}, 2 \mathrm{H}), 1.66(\mathrm{~d}, J 4.2 \mathrm{~Hz}, 1 \mathrm{H}), 1.71-1.73(\mathrm{~m}, 1 \mathrm{H}), 1.83-1.90(\mathrm{~m}, 1 \mathrm{H}), 2.79(\mathrm{~d}, J 7.3 \mathrm{~Hz}, 1 \mathrm{H}), 3.45$ (d, J $7.3 \mathrm{~Hz}, 1 \mathrm{H}), 6.89(\mathrm{~d}, J 6.7 \mathrm{~Hz}, 1 \mathrm{H}), 7.15(\mathrm{~d}, J 6.7 \mathrm{~Hz}, 1 \mathrm{H}),{ }^{13} \mathrm{C}-\mathrm{NMR}:\left(100 \mathrm{MHz}^{\mathrm{C}} \mathrm{CDCl}_{3}\right)$ 11.25, 21.43, 22.04, 27.31, 32.42, 47.20, 48.83, 52.62, 65.72, 78.81, 127.65, 129.18, 132.18, 138.21, 183.25; Anal. Calcd. for $\mathrm{C}_{17} \mathrm{H}_{23} \mathrm{ClN}_{2} \mathrm{OS} \mathrm{C}, 60.25 ; \mathrm{H}, 6.84 ; \mathrm{N}, 8.27 ; \mathrm{S}, 9.49 ;$ found: $\mathrm{C}, 60.20 ; \mathrm{H}, 6.81 ; \mathrm{N}, 8.29 ; \mathrm{S}, 9.52$. 
1-(3-Hydroxy-4,7,7-trimethylbicyclo[2.2.1]heptan-2yl)-3-(4-methoxyphenyl)thiourea (7d). White solid; 60\% yield; $\mathrm{mp} 169-171{ }^{\circ} \mathrm{C} ;[\alpha]_{\mathrm{D}}^{25}=-1.18\left(\mathrm{c}=0.51, \mathrm{CHCl}_{3}\right) ;{ }^{1} \mathrm{HNMR}:\left(400 \mathrm{MHz}, \mathrm{CDCl}_{3}\right) \delta 0.79(\mathrm{~s}, 3 \mathrm{H}), 1.08(\mathrm{~s}, 3 \mathrm{H}), 1.11(\mathrm{~s}$, 3H), 1.127-1.49 (m, 2H,), $1.59(\mathrm{~d}, J 4.2 \mathrm{~Hz}, 1 \mathrm{H}), 1.69-1.71(\mathrm{~m}, 1 \mathrm{H}), 1.80-1.89(\mathrm{~m}, 1 \mathrm{H}), 2.75(\mathrm{~d}, J 7.3 \mathrm{~Hz}, 1 \mathrm{H}), 3.41$ (d, J $7.3 \mathrm{~Hz}, 1 \mathrm{H}), 3.58(\mathrm{~s}, 3 \mathrm{H}), 6.38(\mathrm{~d}, J 6.7 \mathrm{~Hz}, 1 \mathrm{H}), 6.55(\mathrm{~d}, J 6.7 \mathrm{~Hz}, 1 \mathrm{H}),{ }^{13} \mathrm{C}-\mathrm{NMR}:\left(100 \mathrm{MHz}, \mathrm{CDCl}_{3}\right) 13.7$, 21.41, 21.78, 22.82, 32.23, 47.11, 48.78, 52.58, 55.9, 60.2, 78.74, 127.60, 128.12, 129.8, 138.19, 183.13; Anal. Calcd. for $\mathrm{C}_{18} \mathrm{H}_{26} \mathrm{~N}_{2} \mathrm{O}_{2} \mathrm{~S} \mathrm{C}, 64.64 ; \mathrm{H}, 7.84 ; \mathrm{N}, 8.38 ; \mathrm{S}, 9.59$; found: $\mathrm{C}, 64.60 ; \mathrm{H}, 7.80 ; \mathrm{N}, 8.42 ; \mathrm{S}, 9.69$.

Synthesis of chiral diamine 10. According to our previously procedure, ${ }^{46,47}$ the formylation of 2-methyl thiophene was performed according to Vismeier Haack method.

5-Methyl thiophene-2-carbaldehyde $8(5 \mathrm{mmol})$ were dissolved in $10 \mathrm{~mL}$ benzene to which was added (1S,2S)1,2-diphenylethane-1,2-diamine $(5 \mathrm{mmol})$ under nitrogen atmosphere. The mixture was refluxed for $4 \mathrm{~h}$ and water was removed in a Dean-Stark trap. Reaction was controlled by TLC. Imine was concentrated to dryness without purification. The synthesized imine was dissolved in $15 \mathrm{~mL} \mathrm{Et}_{2} \mathrm{O}$ and added to a suspension of 1.40 $\mathrm{mmol} \mathrm{LiAlH}_{4}$ in $10 \mathrm{~mL} \mathrm{Et}{ }_{2} \mathrm{O}$. The mixture was refluxed for $8 \mathrm{~h}$ and controlled by TLC. When the reaction was completed, the mixture was cooled to room temperature and quenched with $15 \mathrm{~mL}$ water. It was extracted with ether $(3 \times 10 \mathrm{~mL})$ and dried over $\mathrm{MgSO}_{4}$. The mixture was filtered and the solvent was evaporated. Crude product 10 were purified by flash column chromatography(EtOAc-hexane, 1:1)

(1S,2S)-N-((5-Methylthiophen-2-yl)methyl)-1,2-diphenylethane-1,2-diamine (10). Brown oil, 80\% yield; $\mathrm{R}_{\mathrm{f}}: 0.15$ (EtOAc:hexane, 1:1); $[\alpha]_{\mathrm{D}}^{25}=-1.41\left(\mathrm{c}=0.38, \mathrm{CHCl}_{3}\right) ;{ }^{1} \mathrm{H} \mathrm{NMR}\left(\mathrm{CDCl}_{3}, 400 \mathrm{MHz}\right) \delta 2.30(\mathrm{~s}, 3 \mathrm{H}), 3.86(\mathrm{~d}, 1 \mathrm{H}$, J $14.4 \mathrm{~Hz}, 1 \mathrm{H}), 3.91(\mathrm{~d}, 1 \mathrm{H}, J 14.4 \mathrm{~Hz}, 1 \mathrm{H}), 4.53(\mathrm{~d}, J$ 4.5, $1 \mathrm{H}), 4.55(\mathrm{~d}, \mathrm{~J} 4.5 \mathrm{HH}$ ), $6.59(\mathrm{~d}, J 3.6 \mathrm{~Hz}, 1 \mathrm{H}), 6.74(\mathrm{~d}, J$ $3.60 \mathrm{~Hz}, 1 \mathrm{H}), 7.156-7.33(\mathrm{~m}, 10 \mathrm{H}, \mathrm{ArH}), 7.86(\mathrm{~s}, 2 \mathrm{H}) ;{ }^{13} \mathrm{C} \mathrm{NMR}\left(\mathrm{CDCl}_{3}, 100 \mathrm{MHz}\right) \delta 15.72,48.23,60.74,67.08$, 105.97, 108.06, 126.9, 127.02, 128.1, 128.5, 128.8, 138.52, 143.76. Anal. Calcd. for $\mathrm{C}_{20} \mathrm{H}_{22} \mathrm{~N}_{2} \mathrm{~S} \mathrm{C}, 74.49 ; \mathrm{H}, 6.88$; N, 8.69; S, 9.94; found: C, 74.52; H, 6.85; N, 8.70; S, 9.93.

Synthesis of thiourea derivatives (11a-d). To solution of chiral compound 10 (1.0 mmol) in $\mathrm{CH}_{2} \mathrm{Cl}_{2}(2.0 \mathrm{~mL})$ was added the corresponding solution of isothiocyanate $(1.1 \mathrm{mmol})$ in $\mathrm{CH}_{2} \mathrm{Cl}_{2}(2.0 \mathrm{~mL})$ at room temperature and reaction mixture was allowed to stir at same temperature until reaction completed (monitoring by TLC). The solvent removed under reduced pressure and the the residue was purified by column chromatography (petroleum ether/ethyl acetate).

1-((1S,2S)-2-((5-Methylthiophen-2-yl)methylamino)-1,2-diphenylethyl)-3-phenylthiourea (11a). White solid; $65 \%$ yield; $\mathrm{mp} 182-184{ }^{\circ} \mathrm{C}$; $[\alpha]_{\mathrm{D}}^{25}=-1.76\left(\mathrm{c}=0.25, \mathrm{CHCl}_{3}\right) ;{ }^{1} \mathrm{H} \mathrm{NMR}:\left(\mathrm{CDCl}_{3}, 400 \mathrm{MHz}\right) \delta 2.35(\mathrm{~s}, 3 \mathrm{H}), 3.88(\mathrm{~d}, 1 \mathrm{H}, \mathrm{J}$ $14.4 \mathrm{~Hz}, 1 \mathrm{H}), 3.93(\mathrm{~d}, 1 \mathrm{H}, J 14.4 \mathrm{~Hz}, 1 \mathrm{H}) 4.55(\mathrm{~d}, J 2.8,1 \mathrm{H}), 4.57(\mathrm{~d}, J 2.8,1 \mathrm{H}), 6.52(\mathrm{~d}, J 3.6 \mathrm{~Hz}, 1 \mathrm{H}), 6.64(\mathrm{~d}, J$ $3.6 \mathrm{~Hz}, 1 \mathrm{H}), 6.95-7.33(\mathrm{~m}, 15 \mathrm{H}, \mathrm{ArH}),{ }^{13} \mathrm{C} \mathrm{NMR}\left(\mathrm{CDCl}_{3}, 100 \mathrm{MHz}\right) \delta 15.72,48.23,62.74,67.08,125.46,126.03$, 126.92, 127.02, 127.28, 128.0, 128.6, 138.45, 143.52, 181.42; Anal. Calcd. for $\mathrm{C}_{27} \mathrm{H}_{27} \mathrm{~N}_{3} \mathrm{~S} C, 70.86 ; \mathrm{H}, 5.95 ; \mathrm{N}$, 9.18; S, 14.01; found: C, 70.82; H, 5.97; N, 9.21; S, 14.00 .

\section{1-((1S,2S)-2-((5-Methylthiophen-2-yl)methylamino)-1,2-diphenylethyl)-3-(3,5-bis(trifluoromethyl)phenyl)}

thiourea (11b). White solid; $79 \%$ yield; $\mathrm{mp} 183-184^{\circ} \mathrm{C}$; $[\alpha]_{\mathrm{D}}^{25}=-1.26\left(\mathrm{c}=0.62, \mathrm{CHCl}_{3}\right) ;{ }^{1} \mathrm{H} \mathrm{NMR:}(\mathrm{CDCl} 3,400 \mathrm{MHz})$ $\delta 2.38(\mathrm{~s}, 3 \mathrm{H}), 3.93(\mathrm{~d}, 1 \mathrm{H}, J 14.4 \mathrm{~Hz}, 1 \mathrm{H}), 3.96(\mathrm{~d}, 1 \mathrm{H}, J 14.4 \mathrm{~Hz}, 1 \mathrm{H}) 4.61(\mathrm{~d}, J 2.8,1 \mathrm{H}), 4.65(\mathrm{~d}, J 2.8,1 \mathrm{H}), 6.59$ (d, J $3.6 \mathrm{~Hz}, 1 \mathrm{H}), 6.72$ (d, J $3.6 \mathrm{~Hz}, 1 \mathrm{H}), 7.10$ (broad s, 2H), 7.25 (s, Ar-H, 1H), 7.32-7.42 (m, 10H); ${ }^{13} \mathrm{C} \mathrm{NMR}$ $\left(\mathrm{CDCl}_{3}, 100 \mathrm{MHz}\right) \delta 15.92,48.29,61.54,67.73,120.14,125.53,126.23,127.45-128.56,132.61,137.23,138.23$, 
138.55, 138.79, 144.57, 181.57; Anal. Calcd. For $\mathrm{C}_{29} \mathrm{H}_{25} \mathrm{~F}_{6} \mathrm{~N}_{3} \mathrm{~S}_{2} \mathrm{C}, 58.67 ; \mathrm{H}, 4.24 ; \mathrm{F}, 19.20 ; \mathrm{N}, 7.08 ; \mathrm{S}, 10.80$; found: $\mathrm{C}, 58.64 ; \mathrm{H}, 4.27 ; \mathrm{N}, 7.05 ; \mathrm{S}, 10.80$.

\section{1-((1S,2S)-2-((5-Methylthiophen-2-yl)methylamino)-1,2-diphenylethyl)-3-(4-chlorophenyl)thiourea}

(11c).

White solid; $63 \%$ yield; $\mathrm{mp} 179-181{ }^{\circ} \mathrm{C}$; $[\alpha]_{\mathrm{D}}^{25}=-1.87\left(\mathrm{c}=0.35, \mathrm{CHCl}_{3}\right) ;{ }^{1} \mathrm{H} \mathrm{NMR:}\left(\mathrm{CDCl}_{3}, 400 \mathrm{MHz}\right) \delta 2.36(\mathrm{~s}, 3 \mathrm{H})$, $3.94(\mathrm{~d}, 1 \mathrm{H}, J 14.4 \mathrm{~Hz}, 1 \mathrm{H}), 3.98(\mathrm{~d}, 1 \mathrm{H}, J 14.4 \mathrm{~Hz}, 1 \mathrm{H}) 4.63(\mathrm{~d}, J 2.9,1 \mathrm{H}), 4.67(\mathrm{~d}, J 2.9,1 \mathrm{H}), 6.57(\mathrm{~d}, J 3.6 \mathrm{~Hz}$, $1 \mathrm{H}), 6.68(\mathrm{~d}, J 3.6 \mathrm{~Hz}, 1 \mathrm{H}), 6.82(\mathrm{~d}, J 8.7 \mathrm{~Hz}, 2 \mathrm{H}), 7.17(\mathrm{~d}, J 8.7 \mathrm{~Hz}, 2 \mathrm{H}), 7.35-7.59(\mathrm{~m}, 10 \mathrm{H}) .{ }^{13} \mathrm{C} \mathrm{NMR}\left(\mathrm{CDCl}_{3}, 100\right.$ $\mathrm{MHz}) \delta 15.8248 .45,61.68,67.89,125.89,126.12,126.89,127.12,127.25,127.98,128.03,128.65,129.12$, 131.12, 135.27, 136.45, 138.35, 138.57, 143.59, 181.49; Anal. Calcd. for $\mathrm{C}_{27} \mathrm{H}_{269} \mathrm{ClN}_{3} \mathrm{~S}_{2} \mathrm{C}, 65.90 ; \mathrm{H}, 5.33 ; \mathrm{N}$, 8.54; S, 13.03; found: C, 65.92; H, 5.36; N, 8.55; S, 12.95 .

1-((1S,2S)-2-((5-Methylthiophen-2-yl)methylamino)-1,2-diphenylethyl)-3-(4-methoxyphenyl)thiourea (11d). White solid; $60 \%$ yield; $\mathrm{mp} 173-175^{\circ} \mathrm{C}$; $[\alpha]_{\mathrm{D}}^{25}=-1.27\left(\mathrm{c}=0.53, \mathrm{CHCl}_{3}\right) ;{ }^{1} \mathrm{H} \mathrm{NMR}:\left(\mathrm{CDCl}_{3}, 400 \mathrm{MHz}\right) 2.30(\mathrm{~s}, 3 \mathrm{H})$, $3.90(\mathrm{~d}, 1 \mathrm{H}, J 14.4 \mathrm{~Hz}, 1 \mathrm{H}), 3.95(\mathrm{~d}, 1 \mathrm{H}, J 14.4 \mathrm{~Hz}, 1 \mathrm{H}) 4.50(\mathrm{~d}, J 6.0,1 \mathrm{H}), 4.62(\mathrm{~d}, J 6.0,1 \mathrm{H}), 6.42(\mathrm{~d}, J 3.6 \mathrm{~Hz}$, $1 \mathrm{H}), 6.58(\mathrm{~d}, J 3.6 \mathrm{~Hz}, 1 \mathrm{H}), 6.67(\mathrm{~d}, J 6.8 \mathrm{~Hz}, 1 \mathrm{H}), 6.99(\mathrm{~d}, J 6.8 \mathrm{~Hz}, 1 \mathrm{H}), 7.10-7.23(\mathrm{~m}, \mathrm{Ar}-\mathrm{H}, 10 \mathrm{H}) ;{ }^{13} \mathrm{C} \mathrm{NMR}$ $\left(\mathrm{CDCl}_{3}, 100 \mathrm{MHz}\right) \delta 15.95,48.45,55.82,61.68,67.89,125.28,125.92,126.02,126.82,127.15,127.32,127.82$, $128.02,128.72,129.46,136.27,138.36,138.57,143.45,156.72,182.27$; Anal. Calcd. for $\mathrm{C}_{28} \mathrm{H}_{29} \mathrm{~N}_{3} \mathrm{OS}_{2} \mathrm{C}, 68.96$; H, 5.99; N, 8.62; S, 13.15; found: C, 68.92; H, 5.97; N, 8.64; S, 13.22 .

Enantioselective intermolecular Morita-Baylis-Hillman reaction. A $25 \mathrm{~mL}$ three-necked vessel was dried under vacuum by applying the Schlenk technique. To soluition of chiral ligand in $5 \mathrm{~mL} \mathrm{CH}_{2} \mathrm{Cl}_{2}$ was added 2nitrobenzaldehyde $(0.015 \mathrm{mmol})$, and methyl vinyl ketone $(0.029 \mathrm{mmol})$. The resulting mixture was stirred until complete loss of starting material was observed by TLC (2:1 hexane/ethyl acetate). The reaction mixture was diluted with chloroform and purification via silica gel chromatography $\left(\mathrm{SiO}_{2}, \mathrm{CHCl}_{3}\right)$ afforded the corresponding products $\mathbf{3}$. Compounds $\mathbf{3} \mathbf{a}-\mathbf{i}$ are known compounds; they were characterized by comparing their ${ }^{1} \mathrm{H},{ }^{13} \mathrm{C}$ NMR spectra with those published in the literature. ${ }^{23,30,48}$ The ee values were determined by HPLC analysis with a chiral column.

(R)-3-[Hydroxy-(4-nitrophenyl)-methyl]-but-3-en-2-one (3a). ${ }^{23} 72 \%$ yield, 92\% ee, ${ }^{1} \mathrm{H} \mathrm{NMR}\left(\mathrm{CDCl}_{3}, 400 \mathrm{MHz}\right)$ : $\delta 2.38(\mathrm{~s}, 3 \mathrm{H}), 3.35(\mathrm{~d}, J=5.6 \mathrm{~Hz}, 1 \mathrm{H}, \mathrm{OH}), 5.71(\mathrm{~d}, J 5.6 \mathrm{~Hz}, 1 \mathrm{H}), 6.06(\mathrm{~s}, 1 \mathrm{H}), 6.30(\mathrm{~s}, 1 \mathrm{H}), 7.58(\mathrm{~d}, J 8.8 \mathrm{~Hz}, 2 \mathrm{H})$, $8.22(\mathrm{~d}, J=8.8 \mathrm{~Hz}, 2 \mathrm{H}) ;{ }^{13} \mathrm{C}-\mathrm{NMR}\left(\mathrm{CDCl}_{3}, 100 \mathrm{MHz}\right): \delta 26.3,72.4,123.8,127.5,127.9,147.7,149.4,149.7,199.6$, HPLC (OD-H column, $\lambda=254 \mathrm{~nm}$, eluent: hexane/2-propanol=95/5, flow rate: $1.0 \mathrm{~mL} / \mathrm{min}$ ): $t_{\mathrm{R}}=28.7 \mathrm{~min}$ (major), $32.4 \mathrm{~min}$ (minor).

(R)-3-[Hydroxy-(2-nitrophenyl)-methyl]-but-3-en-2-one (3b). ${ }^{23} 78 \%$ yield, 84\% ee, $[\alpha]_{D}=-151.0\left(c 0.5, \mathrm{CHCl}_{3}\right)$; ${ }^{1} \mathrm{H} \mathrm{NMR}\left(\mathrm{CDCl}_{3}, 400 \mathrm{MHz}\right): \delta 2.41(\mathrm{~s}, 3 \mathrm{H}), 3.50(\mathrm{~s}, 1 \mathrm{H}), 5.82(\mathrm{~s}, 1 \mathrm{H}), 6.19(\mathrm{~s}, 1 \mathrm{H}), 6.25(\mathrm{~s}, 1 \mathrm{H}), 7.49(\mathrm{t}, J 7.6 \mathrm{~Hz}$, $1 \mathrm{H}), 7.69$ (t, J $7.6 \mathrm{~Hz}, 1 \mathrm{H}), 7.81(\mathrm{~d}, J 8.0 \mathrm{~Hz}, 1 \mathrm{H}), 7.97$ (d, J $8.0 \mathrm{~Hz}, 1 \mathrm{H} ;{ }^{13} \mathrm{C}-\mathrm{NMR}\left(\mathrm{CDCl}_{3}, 100 \mathrm{MHz}\right): \delta 25.4,67.9$, 124.8, 126.4, 128.9, 129.4, 133.7, 136.94, 148.5, 149.3, 201.2; HPLC (AD-H column, $\lambda=254 \mathrm{~nm}$, eluent: hexane/2-propanol=90/10, flow rate: $0.7 \mathrm{~mL} / \mathrm{min}$ ): $t_{\mathrm{R}}=25.7 \mathrm{~min}$ (minor), $28.4 \mathrm{~min}$ (major).

(R)-3-[Hydroxy-(3-nitrophenyl)-methyl]-but-3-en-2-one (3c). ${ }^{48} 68 \%$ yield, 89\% ee; ${ }^{1} \mathrm{H} \mathrm{NMR}\left(\mathrm{CDCl}_{3}, 400 \mathrm{MHz}\right)$ : $\delta 2.39(\mathrm{~s}, 3 \mathrm{H}), 3.34(\mathrm{~s}, 1 \mathrm{H}), 5.69(\mathrm{~s}, 1 \mathrm{H}), 6.11(\mathrm{~s}, 1 \mathrm{H}), 6.31(\mathrm{~s}, 1 \mathrm{H}), 7.54(\mathrm{t}, J 8.0 \mathrm{~Hz}, 1 \mathrm{H}), 7.75(\mathrm{~d}, J 7.6 \mathrm{~Hz}, 1 \mathrm{H})$, $8.17(\mathrm{~d}, J 8.4 \mathrm{~Hz}, 1 \mathrm{H}), 8.25(\mathrm{~s}, 1 \mathrm{H}) ;{ }^{13} \mathrm{C}-\mathrm{NMR}\left(\mathrm{CDCl}_{3}, 100 \mathrm{MHz}\right): \delta$ 26.7, 72.8, 121.9, 122.8, 127.7, 129.8, 132.9, 143.4, 148.8, 149.3, 200.7; HPLC (AD-H column, $\lambda=254 \mathrm{~nm}$, eluent: hexane/2-propanol=90/10, flow rate: 0.8 $\mathrm{mL} / \mathrm{min}$ ): $t_{\mathrm{R}}=21.4 \mathrm{~min}$ (minor), $24.7 \mathrm{~min}$ (major).

(R)-3-[Hydroxy-(4-cyanophenyl)-methyl]-but-3-en-2-one (3d). ${ }^{30} 65 \%$ yield, 87\% ee; $\left.{ }^{1} \mathrm{H} \mathrm{NMR} \mathrm{(CDCl}, 400 \mathrm{MHz}\right)$ : $\delta 2.37(\mathrm{~s}, 3 \mathrm{H}), 3.34(\mathrm{~s}, 1 \mathrm{H}), 5.65(\mathrm{~s}, 1 \mathrm{H}), 6.08(\mathrm{~s}, 1 \mathrm{H}), 6.29(\mathrm{~s}, 1 \mathrm{H}), 7.51(\mathrm{~d}, J 7.6 \mathrm{~Hz}, 2 \mathrm{H}), 7.65(\mathrm{~d}, J 7.6 \mathrm{~Hz}, 2 \mathrm{H})$; 
HPLC (AS-H column, $\lambda=220 \mathrm{~nm}$, eluent: hexane/2-propanol $=85 / 15$, flow rate: $1.0 \mathrm{~mL} / \mathrm{min}$ ): $t_{\mathrm{R}}=21.4 \mathrm{~min}$ (major), $27.6 \mathrm{~min}$ (minor).

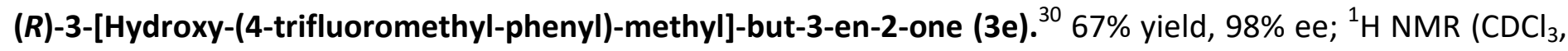
$400 \mathrm{MHz}$ ): $\delta 2.37(\mathrm{~s}, 3 \mathrm{H}), 3.38(\mathrm{~d}, J 5.4 \mathrm{~Hz}, 1 \mathrm{H}), 5.69(\mathrm{~d}, J 5.4 \mathrm{~Hz}, 1 \mathrm{H}), 6.04(\mathrm{~d}, J 1.2 \mathrm{~Hz}, 1 \mathrm{H}), 6.28(\mathrm{~s}, 1 \mathrm{H}), 7.51(\mathrm{~d}$, J $8.0 \mathrm{~Hz}, 2 \mathrm{H}$ ), 7.62 (d, J $8.0 \mathrm{~Hz}, 2 \mathrm{H}$ ); HPLC (AS-H column, $\lambda=220 \mathrm{~nm}$, eluent: hexane/2-propanol = 90/10, flow rate: $1.0 \mathrm{~mL} / \mathrm{min}$ ): $t_{\mathrm{R}}=8,9 \mathrm{~min}$ (major), $10,9 \mathrm{~min}$ (minor).

(R)-3-[Hydroxy-(4-chlorophenyl)-methyl]-but-3-en-2-one (3f). ${ }^{30} 55 \%$ yield, $90 \%$ ee; $\left.{ }^{1} \mathrm{H} \mathrm{NMR} \mathrm{CDCl}_{3}, 400 \mathrm{MHz}\right)$ : $\delta 2.38(\mathrm{~s}, 3 \mathrm{H}), 3.17(\mathrm{~d}, J 5.2 \mathrm{~Hz}, 1 \mathrm{H}), 5.60(\mathrm{~d}, J=5.2 \mathrm{~Hz}, 1 \mathrm{H}), 601(\mathrm{~d}, J 1.1 \mathrm{~Hz}, 1 \mathrm{H}), 6.25(\mathrm{~s}, 1 \mathrm{H}), 7.33(\mathrm{~s}, 4 \mathrm{H}) ;{ }^{13} \mathrm{C}-$ $\mathrm{NMR}\left(\mathrm{CDCl}_{3}, 100 \mathrm{MHz}\right): \delta$ 26.9, 72.9, 127.19, 128.3, 128.9, 133.8, 141.2, 150.0, 200.9; HPLC AS-H column, $\lambda=254 \mathrm{~nm}$, eluent: hexane $/ 2$-propanol $=85 / 15$, flow rate: $1.0 \mathrm{~mL} / \mathrm{min}$ ): $t_{\mathrm{R}}=9.5 \mathrm{~min}$ (major), $11.7 \mathrm{~min}$ (minor).

$(\boldsymbol{R})$-3-[Hydroxy-(2-chlorophenyl)-methyl]-but-3-en-2-one (3g). ${ }^{30} 65 \%$ yield, $85 \%$ ee; ${ }^{1} \mathrm{H} \mathrm{NMR}\left(\mathrm{CDCl}_{3}, 400 \mathrm{MHz}\right)$ : $\delta 2.43(\mathrm{~s}, 3 \mathrm{H}), 3.47(\mathrm{~d}, J 4.5 \mathrm{~Hz}, 1 \mathrm{H}), 5.69(\mathrm{~s}, 1 \mathrm{H}), 6.03(\mathrm{~d}, J 4.5 \mathrm{~Hz}, 1 \mathrm{H}), 6.22(\mathrm{~s}, 1 \mathrm{H}), 7.21-7.26(\mathrm{~m}, 1 \mathrm{H}), 7.31-7.39$ $(\mathrm{m}, 2 \mathrm{H}), 7.59-7.63(\mathrm{~m}, 1 \mathrm{H})$; HPLC (AD-H column, $\lambda=220 \mathrm{~nm}$, eluent: hexane/2-propanol =90/10, flow rate: 1.0 $\mathrm{mL} / \mathrm{min}$ ): $t_{\mathrm{R}}=21.4 \mathrm{~min}$ (minor), $24.7 \mathrm{~min}$ (major).

(R)-3-[Hydroxy-(4-bromophenyl)-methyl]-but-3-en-2-one (3h). ${ }^{30} 58 \%$ yield, 89\% ee, ${ }^{1} \mathrm{H}$ NMR $\left(\mathrm{CDCl}_{3}, 400\right.$ $\mathrm{MHz}): \delta 2.37(\mathrm{~s}, 3 \mathrm{H}), 3.18(\mathrm{~d}, J 5.2 \mathrm{~Hz}, 1 \mathrm{H}), 5.61(\mathrm{~d}, J 5.2 \mathrm{~Hz}, 1 \mathrm{H}), 5.96(\mathrm{~s}, 1 \mathrm{H}), 6.25(\mathrm{~s}, 1 \mathrm{H}), 7.27(\mathrm{~d}, J 8.4 \mathrm{~Hz}, 2 \mathrm{H})$, 7.49 (d, J $8.4 \mathrm{~Hz}, 2 \mathrm{H}$ ); HPLC (AS-H column, $\lambda=220 \mathrm{~nm}$, eluent: hexane/2-propanol = 95/5, flow rate: 1.0 $\mathrm{mL} / \mathrm{min}$ ): $t_{\mathrm{R}}=18.7 \mathrm{~min}$ (major), $25.7 \mathrm{~min}$ (minor).

(R)-3-[Hydroxy-phenylmethyl]-but-3-en-2-one (3j). ${ }^{30} 62 \%$ yield, 78\% ee; $\left.{ }^{1} \mathrm{H} \mathrm{NMR} \mathrm{(CDCl}, 400 \mathrm{MHz}\right): \delta 2.38(\mathrm{~s}$, 3H), $3.14(\mathrm{~d}, \mathrm{~J} 4.4 \mathrm{~Hz}, 1 \mathrm{H}), 5.65(\mathrm{~d}, \mathrm{~J} 4.8 \mathrm{~Hz}, 1 \mathrm{H}), 6.05(\mathrm{~s}, 1 \mathrm{H}),, 6.25(\mathrm{~s}, 1 \mathrm{H}), 7.32-7.41-(\mathrm{m}, 5 \mathrm{H}) ;{ }^{13} \mathrm{C}-\mathrm{NMR}\left(\mathrm{CDCl}_{3}\right.$, $100 \mathrm{MHz}$ ): $\delta$ 26.9, 73.4, 126.1, 126.9, 127.7, 128.6, 141.8, 151.4, 200.8; HPLC (AD-H column, $\lambda=220 \mathrm{~nm}$, eluent: hexane/2-propanol=95/5, flow rate: $0.8 \mathrm{~mL} / \mathrm{min}$ ): $t_{\mathrm{R}}=21.8 \mathrm{~min}$ (minor), $25.7 \mathrm{~min}$ (major).

4-hydroxy-4-(4-methoxyphenyl)-3-methylenebutan-2-one (3i). ${ }^{30} 62 \%$ yield, 55\% ee; ${ }^{1} \mathrm{H}-\mathrm{NMR}\left(\mathrm{CDCl}_{3}, 400\right.$ $\mathrm{MHz}$ ): $\delta 2.38(\mathrm{~s}, 3 \mathrm{H}), 3.81(\mathrm{~s}, 3 \mathrm{H}), 5.62(\mathrm{~d}, J 3.9 \mathrm{~Hz}, 1 \mathrm{H}), 5.74(\mathrm{br}, 1 \mathrm{H}), 6.11(\mathrm{~s}, 1 \mathrm{H}),, 6.27(\mathrm{~s}, 1 \mathrm{H}), 6.91(\mathrm{~d}, J 9.0 \mathrm{~Hz}$, $2 \mathrm{H}$ ), 7.29 ( $\mathrm{d}, J 9.0 \mathrm{~Hz}, 2 \mathrm{H}$ ); HPLC (AD-H column, $\lambda=254 \mathrm{~nm}$, eluent: hexane/2-propanol=90/10, flow rate: 0.8 $\mathrm{mL} / \mathrm{min}$ ): $t_{\mathrm{R}}=17.5 \mathrm{~min}$ (minor), $18.4 \mathrm{~min}$ (major).

Enantioselective intramoleculer Morita-Baylis-Hillman reaction. To a solution of chiral thiourea derivative $\left(0.02 \mathrm{mmol}\right.$, in $\mathrm{CH}_{2} \mathrm{Cl}_{2}(1.0 \mathrm{~mL})$ was added substrates $8(0.2 \mathrm{mmol})$ at room temperature. The reaction mixture was stirred at room temperature until the reaction completed (monitoring by TLC). The solvent was removed under reduced pressure and the residue was purified by a flash column chromatography to afford the intramolecular Baylis-Hillman adducts. Compounds 13a-k are known compounds; they were characterized by comparing their ${ }^{1} \mathrm{H},{ }^{13} \mathrm{C} N M R$ spectra with those published in the literature ${ }^{18,19,21,25}$. The ee values were determined by HPLC analysis with a chiral column. ${ }^{30,41,46,48}$

(R)-(6-Hydroxycyclohex-1-enyl)(phenyl) methanone (13a). ${ }^{46,48} 87 \%$ yield, $83 \%$ ee; $\left.{ }^{1} \mathrm{H}-\mathrm{NMR}\left(400 \mathrm{MHz}^{\mathrm{CDCl}}\right)\right)$ : ठ 1.67-1.71 (m, 1H), 1.85-1.99 (m, 3H), 2.25-2.41 (m, 2H), 3.53 (d, J 1.2 Hz, 1H), $4.79(\mathrm{~d}, J 1.2 \mathrm{~Hz}, 1 \mathrm{H}), 6.79(\mathrm{t}, J$ $4.0 \mathrm{~Hz}, 1 \mathrm{H}), 7.45(\mathrm{t}, J 7.2 \mathrm{~Hz}, 2 \mathrm{H}), 7.57(\mathrm{t}, J 7.2 \mathrm{~Hz}, 1 \mathrm{H}), 7.68$ (d, J $7.2 \mathrm{~Hz}, 2 \mathrm{H}$ ); HPLC analysis (OD-H column, $\lambda=254 \mathrm{~nm}$, eluent: hexane/2-propanol=95/5, flow rate: $1.0 \mathrm{~mL} / \mathrm{min}$ ): $t_{R}=9.8 \mathrm{~min}$ (major), $11.7 \mathrm{~min}$ (minor).

(R)-(6-hydroxycyclohex-1-enyl)(4-nitrophenyl)methanone (13b). ${ }^{46,48} 63 \%$ yield, 95\% ee; ${ }^{1} \mathrm{H}-\mathrm{NMR}(400 \mathrm{MHz}$, $\left.\mathrm{CDCl}_{3}\right): \delta 1.68-1.73(\mathrm{~m}, 1 \mathrm{H}), 1.85-1.99(\mathrm{~m}, 3 \mathrm{H}), 2.25-2.43(\mathrm{~m}, 2 \mathrm{H}), 3.39(\mathrm{~s}, 1 \mathrm{H}),(\mathrm{br} \mathrm{s}, 1 \mathrm{H}), 4.80(\mathrm{~s}, 1 \mathrm{H}), 6.75(\mathrm{~s}$, $1 \mathrm{H}), 7.81(\mathrm{~d}, J 8.4 \mathrm{~Hz}, 2 \mathrm{H}), 8.12(\mathrm{~d}, J 8.4 \mathrm{~Hz}, 2 \mathrm{H})$; HPLC analysis (AD-H column, $\lambda=254 \mathrm{~nm}$, eluent: hexane/2propanol=90/10, flow rate: $0.9 \mathrm{~mL} / \mathrm{min}$ ): $\mathrm{t}_{\mathrm{R}}=21.5 \mathrm{~min}$ (minor), $43.8 \mathrm{~min}$ (major).

(R)-(2-Bromophenyl)(6-hydroxycyclohex-1-enyl)methanone (13c). ${ }^{46,48} 90 \%$ yield, 75\% ee; ${ }^{1} \mathrm{H}-\mathrm{NMR}(400 \mathrm{MHz}$, $\left.\mathrm{CDCl}_{3}\right): \delta 1.62-1.73(\mathrm{~m}, 1 \mathrm{H}) ; 1.78-1.99(\mathrm{~m}, 3 \mathrm{H}), 2.17-2.39(\mathrm{~m}, 2 \mathrm{H}), 3.38(\mathrm{~s}, 1 \mathrm{H}), 4.82(\mathrm{t}, J 4.4 \mathrm{~Hz}, 1 \mathrm{H}), 6.63(\mathrm{t}, J$ 
$4.0 \mathrm{~Hz}, 1 \mathrm{H}), 7.27-7.41(\mathrm{~m}, 3 \mathrm{H}), 7.62(\mathrm{~d}, J 8.0 \mathrm{~Hz}, 1 \mathrm{H}) ;{ }^{13} \mathrm{C}-\mathrm{NMR}\left(100 \mathrm{MHz}, \mathrm{CDCl}_{3}\right): \delta$ 17.9, 26.3, 29.9, 63.5, 120.1, 127.5, 128.9, 130.7, 133.3, 140.9, 141.76, 150.9, 198.9; HPLC analysis (AS-H column, $\lambda=254 \mathrm{~nm}$, eluent: hexane/2-propanol=90/10, flow rate: $1.0 \mathrm{~mL} / \mathrm{min}$ ): $\mathrm{t}_{\mathrm{R}}=9.5 \mathrm{~min}$ (major), $18.42 \mathrm{~min}$ (minor).

(R)-(3-Bromophenyl)(6-hydroxycyclohex-1-enyl)methanone (13d). ${ }^{46,48} 90 \%$ yield, 78\% ee; ${ }^{1} \mathrm{H}-\mathrm{NMR}(400 \mathrm{MHz}$, $\left.\mathrm{CDCl}_{3}\right): \delta 1.65-1.71(\mathrm{~m}, 1 \mathrm{H}), 1.82-1.99(\mathrm{~m}, 3 \mathrm{H}), 2.25-2.45(\mathrm{~m}, 2 \mathrm{H}), 3.39(\mathrm{~s}, 1 \mathrm{H}), 4.77(\mathrm{~s}, 1 \mathrm{H}), 6.75(\mathrm{t}, J 4.0 \mathrm{~Hz}, 1 \mathrm{H})$, $7.38(\mathrm{t}, J 8.0 \mathrm{~Hz}, 1 \mathrm{H}), 7.59(\mathrm{~d}, J 8.0 \mathrm{~Hz}, 1 \mathrm{H}), 7.70(\mathrm{~d}, J 8.0 \mathrm{~Hz}, 1 \mathrm{H}), 7.80(\mathrm{~s}, 1 \mathrm{H}) ;{ }^{13} \mathrm{C}-\mathrm{NMR}\left(100 \mathrm{MHz}^{\mathrm{C} C D C l}\right)_{3}: \delta$ 17.8, 26.7, 29.9, 64.3, 123.1, 127.9, 130.2, 132.6, 134.9, 139.6, 140.3, 148.2, 197.9; HPLC analysis(AD-H column, $\lambda=254 \mathrm{~nm}$, eluent: hexane/2-propanol=90/10, flowrate: $1.0 \mathrm{~mL} / \mathrm{min}$ ): $\mathrm{t}_{\mathrm{R}}=12.6 \mathrm{~min}(\operatorname{minor}), 14.8 \mathrm{~min}$ (major).

(R)-(4-Bromophenyl)(6-hydroxycyclohex-1-enyl)methanone (13e). ${ }^{46,48} 85 \%$ yield, 80\% ee; ${ }^{1} \mathrm{H}-\mathrm{NMR}(400 \mathrm{MHz}$, $\left.\mathrm{CDCl}_{3}\right): \delta 1.65-1.74(\mathrm{~m}, 1 \mathrm{H}), 1.85-12.02(\mathrm{~m}, 3 \mathrm{H}), 2.26-2.42(\mathrm{~m}, 2 \mathrm{H}), 2.59(\mathrm{br} \mathrm{s}, 1 \mathrm{H}), 4.79(\mathrm{~s}, 1 \mathrm{H}), 6.78(\mathrm{t}, J=4.0$ $\mathrm{Hz}, 1 \mathrm{H}), 7.57(\mathrm{~d}, J 8.4 \mathrm{~Hz}, 2 \mathrm{H}), 7.65(\mathrm{~d}, J 8.4 \mathrm{~Hz}, 2 \mathrm{H})$; HPLC analysis (AD-H column, $\lambda=254 \mathrm{~nm}$, eluent: hexane/2propanol=90/10, flow rate: $1.0 \mathrm{~mL} / \mathrm{min}$ ): $\mathrm{t}_{\mathrm{R}}=20.3 \mathrm{~min}$ (minor), $23.7 \mathrm{~min}$ (major).

(R)-(4-Chlorophenyl)(6-hydroxycyclohex-1-enyl)methanone (13f). ${ }^{30,48} 83 \%$ yield, 81\% ee; ${ }^{1} \mathrm{H}-\mathrm{NMR}(400 \mathrm{MHz}$, $\left.\mathrm{CDCl}_{3}\right): \delta 1.65-1.73(\mathrm{~m}, 1 \mathrm{H}), 1.89-1.99-(\mathrm{m}, 3 \mathrm{H}), 2.27-2.45(\mathrm{~m}, 2 \mathrm{H}), 2.54(\mathrm{br} \mathrm{s}, 1 \mathrm{H}), 4.78(\mathrm{~s}, 1 \mathrm{H}), 6.73(\mathrm{t}, J 4.0 \mathrm{~Hz}$, $1 \mathrm{H}), 7.47(\mathrm{~d}, J 8.4 \mathrm{~Hz}, 2 \mathrm{H}), 7.68(\mathrm{~d}, J 8.4 \mathrm{~Hz}, 2 \mathrm{H})$; HPLC analysis (AS-H column, $\lambda=254 \mathrm{~nm}$, eluent: hexane/2propanol=90/10, flow rate: $1.0 \mathrm{~mL} / \mathrm{min}$ ): $t_{R}=8,7 \mathrm{~min}$ (major), $13.5 \mathrm{~min}$ (minor).

(R)-(4-Fluorophenyl)(6-hydroxycyclohex-1-enyl)methanone (13g). ${ }^{30,48} 78 \%$ yield, 80\% ee; ${ }^{1} \mathrm{H}-\mathrm{NMR}(400 \mathrm{MHz}$, $\left.\mathrm{CDCl}_{3}\right): \delta$ 1.65-1.73- $(\mathrm{m}, 1 \mathrm{H}), 1.88-1.99(\mathrm{~m}, 3 \mathrm{H}), 2.21-2.43(\mathrm{~m}, 2 \mathrm{H}), 3.45(\mathrm{~s}, 1 \mathrm{H}), 4.78(\mathrm{~s}, 1 \mathrm{H}), 6.72(\mathrm{t}, J 4.0 \mathrm{~Hz}$, 1H), $7.15(\mathrm{t}, J 8.4 \mathrm{~Hz}, 2 \mathrm{H}), 7.72-7.77(\mathrm{~m}, 2 \mathrm{H}) ;{ }^{13} \mathrm{C}-\mathrm{NMR}\left(100 \mathrm{MHz}, \mathrm{CDCl}_{3}\right): \delta 17.84,26.5,30.3,64.3,115.9,131.4$, 134.4, 141.2, 146.7, 165.6, 197.9; HPLC analysis (AD-H column, $\lambda=254 \mathrm{~nm}$, eluent: hexane/2-propanol=90/10, flow rate: $0.8 \mathrm{~mL} / \mathrm{min}$ ): $\mathrm{t}_{\mathrm{R}}=12.9 \mathrm{~min}$ (minor), $15.0 \mathrm{~min}$ (major).

(R)-(6-Hydroxycyclohex-1-enyl)(o-tolyl)methanone (13h) ${ }^{39}$ 95\% yield, 62\% ee; ${ }^{1} \mathrm{H}-\mathrm{NMR}\left(\mathrm{CDCl}_{3}, 400 \mathrm{MHz}\right) \delta$ 1.59-1.69 (m, 1H), 1.79-1.94 (m, 3H), 2.17-2.35 (m, 2H), $2.32(\mathrm{~s}, 3 \mathrm{H}), 3.55(\mathrm{br} \mathrm{s}, 1 \mathrm{H}), 4.77(\mathrm{br} \mathrm{s}, 1 \mathrm{H}), 6.62(\mathrm{t}, J=$ $4.0 \mathrm{~Hz}, 1 \mathrm{H}), 7.231(\mathrm{~m}, 3 \mathrm{H}), 7.34(\mathrm{~m}, 1 \mathrm{H}) ;{ }^{13} \mathrm{C}-\mathrm{NMR}\left(\mathrm{CDCl}_{3}, 100 \mathrm{MHz}\right) \delta 17.9,19.9,27.0,30.2,642,125.2,127.8$, 130.2, 131.2, 135.9, 139.3, 141.9, 149.1, 202.1; HPLC analysis (Chiralcel OD column, $\lambda=254 \mathrm{~nm}$, eluent; hexanes: ethanol=98/2, flow rate; $0.75 \mathrm{~mL} / \mathrm{min}$; $t_{R}=13.4 \mathrm{~min}$ (major), $27.1 \mathrm{~min}$ (minor).

(R)-(6-Hydroxycyclohex-1-enyl)(p-tolyl)methanone (13i) ${ }^{41} 80 \%$ yield, 65\% ee; ${ }^{1} \mathrm{H}-\mathrm{NMR}\left(400 \mathrm{MHz} \mathrm{CDCl}_{3}\right): \delta$ 1.62-1.68 (m, 1H), 1.86-1.98 (m, 3H), 2.21-2.380 (m, 2H), $2.43(\mathrm{~s}, 3 \mathrm{H}), 2.93(\mathrm{br} \mathrm{s}, 1 \mathrm{H}), 4.75(\mathrm{~s}, 1 \mathrm{H}), 6.73(\mathrm{t}, J 4.0$ $\mathrm{Hz}, 1 \mathrm{H}), 7.26(\mathrm{~d}, J 7.6 \mathrm{~Hz}, 2 \mathrm{H}), 7.59(\mathrm{~d}, J 8.0 \mathrm{~Hz}, 2 \mathrm{H})$; HPLC analysis (AD-H column, $\lambda=254 \mathrm{~nm}$, eluent: hexane/2propanol=90/10, flowrate: $1.0 \mathrm{~mL} / \mathrm{min}$ ): $t_{R}=15.8 \mathrm{~min}$ (major), $17.8 \mathrm{~min}$ (minor).

(R)-(6-Hydroxycyclohex-1-enyl)(4-methoxyphenyl) methanone (13j) ${ }^{41} 78 \%$ yield, 60\% ee; ${ }^{1} \mathrm{H}-\mathrm{NMR}(400 \mathrm{MHz}$, $\left.\mathrm{CDCl}_{3}\right): \delta 1.64-1.70(\mathrm{~m}, 1 \mathrm{H}), 1.86-1.97(\mathrm{~m}, 3 \mathrm{H}), 2.24-2.41(\mathrm{~m}, 2 \mathrm{H}), 3.62(\mathrm{br} \mathrm{s}, 1 \mathrm{H}), 3.91(\mathrm{~s}, 3 \mathrm{H}), 4.73(\mathrm{~s}, 1 \mathrm{H}), 6.68$ $(\mathrm{t}, J 4.0 \mathrm{~Hz}, 1 \mathrm{H}), 6.97(\mathrm{~d}, J 8.8 \mathrm{~Hz}, 2 \mathrm{H}), 7.73(\mathrm{~d}, \mathrm{~J} J 8.8 \mathrm{~Hz}, 2 \mathrm{H}) ;{ }^{13} \mathrm{C} \mathrm{NMR}\left(100 \mathrm{MHz}, \mathrm{CDCl}_{3}\right): \delta 17.5,26.5,30.0$, 55.7, 65.3, 114.1, 130.4, 132.1, 139.9, 145.2, 163.4, 198.8; HPLC analysis (AS-H column, $\lambda=254 \mathrm{~nm}$, eluent: $\mathrm{n}$ hexane/i-propanol=90/10, flow rate: $1.0 \mathrm{~mL} / \mathrm{min}$ ): $\mathrm{t}_{\mathrm{R}}=15.2 \mathrm{~min}$ (major), $31.3 \mathrm{~min}$ (minor);

(R)-(6-Hydroxycyclohex-1-enyl)(thiophen-2-yl)methanone (13k). ${ }^{30,48}$ 65\% yield, 72\% ee; ${ }^{1} \mathrm{H}-\mathrm{NMR}(400 \mathrm{MHz}$, $\left.\mathrm{CDCl}_{3}\right): \delta 1.62-1.71(\mathrm{~m}, 1 \mathrm{H}), 1.79-1.97(\mathrm{~m}, 3 \mathrm{H}), 2.27-2.45(\mathrm{~m}, 2 \mathrm{H}), 2.72(\mathrm{~s}, 1 \mathrm{H}), 4.71(\mathrm{~s}, 1 \mathrm{H}), 6.98(\mathrm{t}, J 4.0 \mathrm{~Hz}, 1 \mathrm{H})$, 7.13-7.19 (m, 1H), $7.65(\mathrm{~d}, J 2.8 \mathrm{~Hz}, 1 \mathrm{H}), 7.71(\mathrm{~d}, J 4.8 \mathrm{~Hz}, 1 \mathrm{H})$; HPLC analysis (AS-H column, $\lambda=254 \mathrm{~nm}$, eluent: hexane/2-propanol=90/10, flow rate: $1.0 \mathrm{~mL} / \mathrm{min}$ ): $\mathrm{t}_{\mathrm{R}}=11.2 \mathrm{~min}$ (major), $18.2 \mathrm{~min}$ (minor). 
This research (08F0201 and 1005M0115) is financially supported by the Scientific Research Commission of Mustafa Kemal University.

\section{References}

1. Basavaiah, D.; Rao, P. D.; Hyma, R. S. Tetrahedron 1996, 52, 8001-8062. https://doi.org/10.1016/0040-4020(96)00154-8

2. Langer, P. Angew. Chem. Int. Ed. 2000, 39, 3049-3052 https://doi.org/10.1002/1521-3773(20000901)39:17<3049::AID-ANIE3049>3.0.CO;2-5

3. Basavaiah, D.; Rao, K. V.; Reddy, R. J. Chem. Soc. Rev. 2007, 36, 1581-1588. https://doi.org/10.1039/b613741p

4. Singh, V.; Batra, S. Tetrahedron 2008, 64, 4511-4574 https://doi.org/10.1016/j.tet.2008.02.087

5. Kurasaki, H.; Okamoto, I.; Morita, N.; Tamura, O. Org. Lett. 2009, 11, 1179-1181. https://doi.org/10.1021/0l900032h

6. Shi, Y.-L.; Xu, Y.-M.; Shi, M. Adv. Synth. Catal. 2004, 346, 1220-1230. https://doi.org/10.1002/adsc.200404045

7. Bugarin, A.; Connell, B. T. J. Org. Chem. 2009, 74, 4638-4641. https://doi.org/10.1021/j0900603w

8. Shi, M.; Liu, X.-G. Org. Lett. 2008, 10, 1043-1046. https://doi.org/10.1021/ol7028806

9. He, L.; Jian, T.-Y.; Ye, S. J. Org. Chem. 2007, 72, 7466-7468. https://doi.org/10.1021/jo071247i

10. Basavaiah, D.; Krishnamacharyulu, M.; Rao, A. J. Synth. Commun. 2000, 30, 2061-2069. https://doi.org/10.1080/00397910008087256

11. Rafel, S.; Leahy, J. W. J. Org. Chem. 1997, 62, 1521-1522. https://doi.org/10.1021/jo961224w

12. Wu, Z.; Zhou, G.; Zhou, J.; Guo, W. Synth. Commun. 2006, 36, 2491-2502. https://doi.org/10.1080/00397910600781273

13.Yu, C.; Hu, L. J. Org. Chem. 2002, 67, 219.-223. https://doi.org/10.1021/jo016004j

14. Morita, K.; Suzuki, Z.; Hirose, H. Bull. Chem. Soc. Jpn. 1968, 41, 2815. https://doi.org/10.1246/bcsj.41.2815 15. Shi, M.; Xu, Y.-M. Angew. Chem. Int. Ed. 2002, 41, 4507-4510. https://doi.org/10.1002/1521-3773(20021202)41:23<4507::AID-ANIE4507>3.0.CO;2-I 16. Yang, K. S.; Lee, W. D.; Pan, J. F.; Chen, K. J. Org. Chem. 2003, 68, 915-919. 


\section{https://doi.org/10.1021/jo026318m}

17. Walsh, L. M.;Winn, C. L.; Goodman, J. M. Tetrahedron Lett. 2002, 43, 8219-8222.

https://doi.org/10.1016/S0040-4039(02)02053-1

18. Yamada, Y. M. A.; Ikegami, S. Tetrahedron Lett. 2000, 41, 2165-2169.

https://doi.org/10.1016/S0040-4039(00)00125-8

19. Barrette, A. G. M.; Cook, A. S.; Kamimura, A. Chem. Commun. 1998, 2533-2534

https://doi.org/10.1039/a806115g

20. Shi, M.; Jiang, J. K. Tetrahedron: Asymmetry 2002, 13,1941-1947.

https://doi.org/10.1016/S0957-4166(02)00485-8

21. Pegot, B.; Vo-Thanh, G.;Gori, D.; Loupy, A. Tetrahedron Lett. 2004, 45, 6425-6428.

https://doi.org/10.1016/j.tetlet.2004.06.134

22. Imbriglio, J. E.; Vasbinder, M. M.; Miller, S. J. Org. Lett. 2003, 5, 3741-3743.

https://doi.org/10.1021/ol035466b

23. Krishna, P. R.; Kannan, V.; Reddy, P. V. N. Adv. Synth. Catal. 2004, 346, 603-606.

https://doi.org/10.1002/adsc.200303217

24. Sohtome, Y.; Tanatani, A.; Hashimoto, Y.; Nagasawa, K. Tetrahedron Lett. 2004, 45, 5589-5592.

https://doi.org/10.1016/j.tetlet.2004.05.137

25. Pegot, B.; Vo-Thanh, G.; Gori, D.; Loupy, A. Tetrahedron Lett. 2004, 45, 6425-6428.

https://doi.org/10.1016/i.tetlet.2004.06.134

26. Markó, I. E.; Giles, P. R.; Hindley, N. J. Tetrahedron 1997, 53, 1015-1024.

https://doi.org/10.1016/S0040-4020(96)01001-0

27. Basavaiah, D.; Rao, A. J.; Satyanarayana, T. Chem. Rev. 2003, 103, 811-891.

https://doi.org/10.1021/cr010043d

28. Barrette, A. G. M.; Dozzo, P.; White, A. J. P.; Williams, D. J. Tetrahedron 2002, 58, 7303-7313.

https://doi.org/10.1016/S0040-4020(02)00761-5

29. Iwabuchi, Y; Nakatani, M; Yokoyama, N; Hatakeyama, S. J. Am. Chem. Soc. 1999, 121, 10219-10220.

https://doi.org/10.1021/ja992655+

30. Hayashi, Y.; Tamura, T.; Shoji, M. Adv. Synth. Catal. 2004, 346, 1106-1110.

https://doi.org/10.1002/adsc.200404069

31. Yuan, K.; Zhang, L.; Song, H.-L.; Hu, Y.; Wu, X.-Y. Tetrahedron Lett. 2008, 49, 6262-6264.

https://doi.org/10.1016/i.tetlet.2008.08.042

32. Wang, J.; Li, H.; Yu, X.; Zu, L.; Wang, W. Org. Lett. 2005, 7, 4293-4296.

https://doi.org/10.1021/ol051822+

33. Yuan, K.; Song, H.-L.; Hu, Y.; Wu, X.-Y. Tetrahedron 2009, 65, 8185-8190.

https://doi.org/10.1016/j.tet.2009.07.066 
34. Gong, J.-J.; Yuan, K.; Wu, X.-Y. Tetrahedron:Asymmetry 2009, 20, 2117-2120.

https://doi.org/10.1016/j.tetasy.2009.07.047

35. Ito, H.; Takenaka, Y.; Fukunishi, S.; Iguchi, K. Synthesis 2005, 3035-3038.

https://doi.org/10.1055/s-2005-916030

36. Shi, M.; liu, Y.-H. Org. Biomol. Chem. 2006, 4, 1468-1470.

https://doi.org/10.1039/b600854b

37. Buono, G.; Chiodi, O.; Wills, M. Synlett 1999, 377-378.

https://doi.org/10.1055/s-1999-2620

38. Aroyan, C. E.; Vasbinder, M. M.; Miller, S. J. Org. Lett. 2005, 7, 3849-3851.

https://doi.org/10.1021/ol0513544

39. Gong, J.-J.; Yuan, K.; Song, H.-L.; Wu, X.-Y. Tetrahedron 2010, 66, 2439-2443.

https://doi.org/10.1016/j.tet.2010.01.085

40. Chen, S.-H.; Hong, B.-C.; Su, C.-F.; Sarshar, S. Tetrahedron Lett. 2005, 46, 8899-8903.

https://doi.org/10.1016/j.tetlet.2005.10.072

41. Seidel, F.; Gladysz, J. Synlett 2007, 986-988.

42. Oliveira, L.F.; Costa V. E. U. Tetrahedron:Asymmetry 2004, 15, 2583-2590.

https://doi.org/10.1016/j.tetasy.2004.07.027

43. Costa, V. E. U.; Lapis, A. A. M.; Kreutz, O. C.; Pohlman, A. R. Tetrahedron:Asymmetry 2001, 12, 557-561. https://doi.org/10.1016/S0957-4166(01)00085-4

44. Chittenden, R. A.; Cooper, G. H. J. Chem Soc. (C) 1970, 49-54.

https://doi.org/10.1039/j39700000049

45. Passi, A. M. B.; Henin, F.; Muzart, J.; Péte, J. P. Bull.Soc. Chim. Fr. 1993, 130, 214-217.

46. Aydin, A. E. Appl. Organomet. Chem. 2013,. 27, 5, 283-289.

https://doi.org/10.1002/aoc.2969

47. Aydin, A. E. Tetrahedron:Asymmetry 2013, 24, 8, 444-448.

https://doi.org/10.1002/aoc.2969

48. Richards, E. L.; Murphy, P. J.; Dinon, F.; Fratucello, S.; Brown, P. M.; Gelbrich, T.; Hursthouse, M. B.

Tetrahedron 2001, 57, 7771-7784

https://doi.org/10.1016/S0040-4020(01)00744-X

This paper is an open access article distributed under the terms of the Creative Commons Attribution (CC BY) license (http://creativecommons.org/licenses/by/4.0/ 